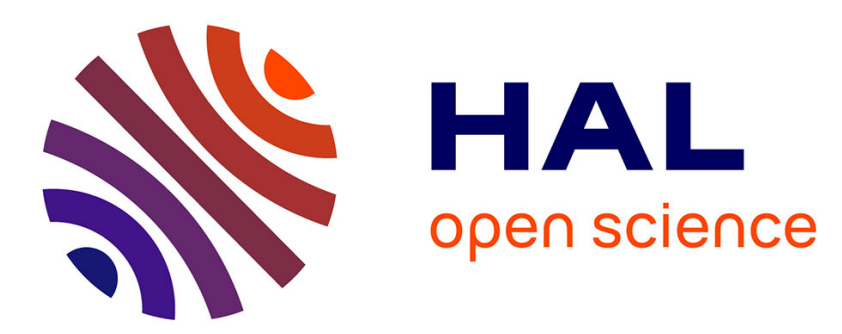

\title{
Analyse bayésienne des courbes de tarage et de leurs incertitudes: la méthode BaRatin
}

J. Le Coz, C. Chaléon, L. Bonnifait, R. Le Boursicaud, Benjamin Renard, F. Branger, J. Diribarne, M. Valente

\section{- To cite this version:}

J. Le Coz, C. Chaléon, L. Bonnifait, R. Le Boursicaud, Benjamin Renard, et al.. Analyse bayésienne des courbes de tarage et de leurs incertitudes: la méthode BaRatin. La Houille Blanche - Revue internationale de l'eau, 2013, 6, pp.31-41. 10.1051/lhb/2013048 . hal-01122602

\section{HAL Id: hal-01122602 \\ https://hal.science/hal-01122602}

Submitted on 4 Mar 2015

HAL is a multi-disciplinary open access archive for the deposit and dissemination of scientific research documents, whether they are published or not. The documents may come from teaching and research institutions in France or abroad, or from public or private research centers.
L'archive ouverte pluridisciplinaire HAL, est destinée au dépôt et à la diffusion de documents scientifiques de niveau recherche, publiés ou non, émanant des établissements d'enseignement et de recherche français ou étrangers, des laboratoires publics ou privés. 


\title{
Analyse bayésienne des courbes de tarage et de leurs incertitudes : la méthode BaRatin
}

\author{
Jérôme LE COZ ${ }^{1}$, Carine CHALÉON ${ }^{2}$, Laurent BONNIFAIT ${ }^{3}$, Raphaël LE BOURSICAUD ${ }^{1}$, \\ Benjamin RENARD ${ }^{1}$, Flora BRANGER ${ }^{1}$, Julien DIRIBARNE² ${ }^{2}$, Marc VALENTE ${ }^{2}$
}

\author{
1. Irstea Lyon, Unité de Recherche Hydrologie-Hydraulique, 5 rue de la Doua CS70077 69626 Villeurbanne cedex, France (jerome.lecoz@irstea.fr) \\ 2. DRIEE Ile-de-France, Pôle hydrologie et prévision des crues, 10 rue de Crillon 75194 Paris Cedex 04, France \\ (carine.chaleon@developpement-durable.gouv.fr) \\ 3. DRIEA / CETE Ile-de-France, DRIEA, Unité Hydrologie - Gestion des eaux pluviales, 12, rue Teisserenc de Bort, 78197 Trappes cedex, France \\ (laurent.bonnifait@developpement-durable.gouv.fr)
}

\begin{abstract}
RÉSUMÉ. - Parmi d'autres méthodes, l'inférence bayésienne a récemment émergé comme un cadre prometteur pour établir les relations hauteur-débit et en estimer l'incertitude associée de façon rigoureuse. Une méthodologie et des outils ont été développés pour permettre l'analyse des courbes de tarage stationnaires, c'est-à-dire sous hypothèse de stabilité de la relation hauteur-débit sur la période considérée (pas de détarage). Les principes de la méthode BaRatin sont présentés à travers ses trois principales étapes : 1) détermination des a priori hydrauliques, 2) critique et validation des jaugeages, 3) inférence bayésienne et simulation d'un faisceau de courbes vraisemblables. L'application opérationnelle de la méthode est facilitée grâce à l'interface graphique BaRatinAGE diffusée librement avec le logiciel BaRatin, et en cours d'intégration dans le logiciel d'hydrométrie BAREME. Le potentiel de la méthode est illustré par un exemple d'application à une station hydrométrique et la démarche d'appropriation par un service d'hydrométrie. Outre l'application à d'autres types de courbes d'étalonnage, la principale perspective de développement est la prise en compte d'effets non-stationnaires à l'aide de paramètres variables dans le temps.
\end{abstract}

Mots-clés : BaRatin, incertitudes, hydrométrie, modélisation

\section{Bayesian analysis of rating curves and their uncertainties: the BaRatin method}

\begin{abstract}
Among other methods, Bayesian inference recently emerged as a promising framework for establishing stage-discharge relationships and for rigorously estimating the associated uncertainty. A methodology and some tools have been developed to analyse stationary rating curves, i.e., assuming that the stage-discharge relationship is stable over the period under consideration (no rating shifts). The principles of the BaRatin method are presented through its three main steps: 1) determination of hydraulic priors, 2) review and validation of streamgaugings, 3) Bayesian inference and simulation of a set of plausible curves. The operational application of the method is made easier through the GUI BaRatinAGE, which is freely distributed along with the BaRatin software and is currently being integrated into the hydrometry software BAREME. The potential of the method is illustrated with an example of application to a gauging station and with the process of appropriation by a hydrometry service. Besides the application to other types of calibration curves, the main development perspective for the BaRatin method is to take into account non-stationary effects with time-varying parameters.
\end{abstract}

Key-words: BaRatin, uncertainty, hydrometry, modelling

\section{INTRODUCTION}

Le plus souvent, les mesures de débit en continu dans les cours d'eau sont fondées sur des relations hauteur-débit simples (ou « courbes de tarage ») construites en utilisant des hypothèses implicites ou explicites sur les contrôles hydrauliques en jeu, et des mesures hauteur-débit ponctuelles appelées « jaugeages ». Ces dernières années, l'application de l'inférence bayésienne et des simulations Monte-Carlo par chaînes de Markov (MCMC) a apporté de nouvelles solutions au problème de l'analyse de l'incertitude des courbes de tarage. À notre connaissance, [Moyeed et Clarke, 2005] ont été les premiers à rapporter l'analyse bayésienne de relations hauteur-débit. D'autres études bayésiennes avec simulation MCMC se sont penchées sur l'incertitude due au nombre limité de jaugeages sur un contrôle hydraulique homogène [Reitan et Petersen-Overleir, 2008], sur l'établissement d'une fonction puissance par morceaux pour une succession de contrôles [Reitan et Petersen-Overleir, 2009], et sur les procédures de tarage de stations soumises à influence aval variable [Petersen-Overleir et Reitan, 2009].

Le cadre bayésien présente des avantages décisifs pour aborder la question. Tout d'abord, la connaissance hydraulique de la station hydrométrique peut être explicitement convertie en distributions a priori des paramètres de la fonction hauteur-débit supposée. Deuxièmement, comme avec n'importe quelle technique de vraisemblance, il est possible de déterminer une fonction de vraisemblance qui tient 
compte de l'incertitude des observations individuelles (ici, les jaugeages), conduisant à des modèles hétéroscédastiques similaires à celui de [Petersen-Overleir, 2004], par exemple. Ainsi, l'information tirée à la fois des observations (fonctions de vraisemblance) et des connaissances (a priori hydrauliques) est utilisée pour produire les résultats sous la forme d'une distribution a posteriori. En outre, comme la technique est directement basée sur les densités de probabilité, l'analyse d'incertitude peut être réalisée d'une manière simple et claire en utilisant des centiles de la distribution a posteriori.

Dans la plupart des études bayésiennes publiées, l'analyse des contrôles hydrauliques n'est pas suffisamment explicitée et très souvent, les exposants des lois hydrauliques obtenues n'ont pas de base physique interprétable avec les formules usuelles. En s'attachant à améliorer l'interprétation des contrôles hydrauliques, la méthodologie BaRatin (pour BAyesian RATINg curve analysis) et les outils informatiques associés ont été développés par Irstea et sont diffusés librement [Branger et al., 2012]. Cette méthode permet l'analyse des courbes de tarage stationnaires, c'est-à-dire sous hypothèse de stabilité de la relation hauteur-débit sur la période considérée (pas de détarage). Dans cet article, nous en présentons les principes et en illustrons l'application opérationnelle.

\section{PRINCIPES DE LA MÉTHODE BARATIN}

La Fig.1 permet de visualiser les principales étapes de la méthode BaRatin, en amont et en aval du solveur BaRatin qui effectue l'inférence bayésienne et les simulations MCMC de courbes de tarage. La première étape est le choix de l'équation de courbe de tarage à partir de la configuration hydraulique du site d'étude et la spécification des a priori sur les paramètres de cette équation, à partir des données topographiques et de simulation numérique lorsque cela est utile, notamment pour l'extrapolation aux forts débits non jaugés. Deuxièmement, l'incertitude de chaque jaugeage doit être quantifiée. Enfin, ces incertitudes sont prises en compte individuellement par le simulateur bayésien BaRatin, qui génère un faisceau de courbes de tarages vraisemblables dont on peut extraire la courbe de tarage la plus probable et l'incertitude associée, au niveau de confiance requis (en général $95 \%$ en hydrométrie, cf. le HUG [ISO/TS 25377, 2007]). Ces trois principales étapes, qui permettent d'évaluer la courbe de tarage et son incertitude sous hypothèse stationnaire (pas de détarage ou évolution de la relation hauteur-débit), sont détaillées ci-dessous. Au-delà, il est possible d'évaluer l'incertitude dans les débits instantanés (" hydrogramme »), en prenant en compte non seulement l'incertitude sur la relation hauteur-débit, mais aussi l'incertitude sur les enregistrements de hauteur d'eau (qui se propage sur les débits proportionnellement à $d Q / d h$, l'inverse du coefficient de sensibilité de la station), les effets de régime transitoire (hystérésis) et la modification des contrôles hydrauliques. Ces aspects, encore en développement, ne seront pas développés dans cet article.

\section{II.1. Détermination des a priori hydrauliques}

\section{II.1.1. Notion de contrôle hydraulique}

Dans la section de cours d'eau où est située la station hydrométrique, la relation entre la hauteur d'eau et le débit est déterminée par des caractéristiques physiques du chenal que l'on appelle " contrôles hydrauliques ». Selon la gamme de débit considérée, les contrôles hydrauliques en jeu peuvent se succéder ou s'ajouter les uns aux autres, comme illustré par l'exemple simple mais typique de la Figure 2. Pour une valeur de débit croissante, le niveau d'eau à cette station fictive est d'abord contrôlé par un petit seuil naturel, qui est ensuite ennoyé et passe alors le relais à un deuxième seuil situé plus en aval et présentant une plus grande chute. Ce deuxième seuil est à son tour ennoyé, le niveau étant alors contrôlé par le chenal du lit mineur. Lorsque le débordement intervient, le contrôle hydraulique par le lit majeur s'ajoute à celui par le lit mineur.

On distingue deux types de contrôles hydrauliques : le contrôle par une section (cas des seuils naturels), et

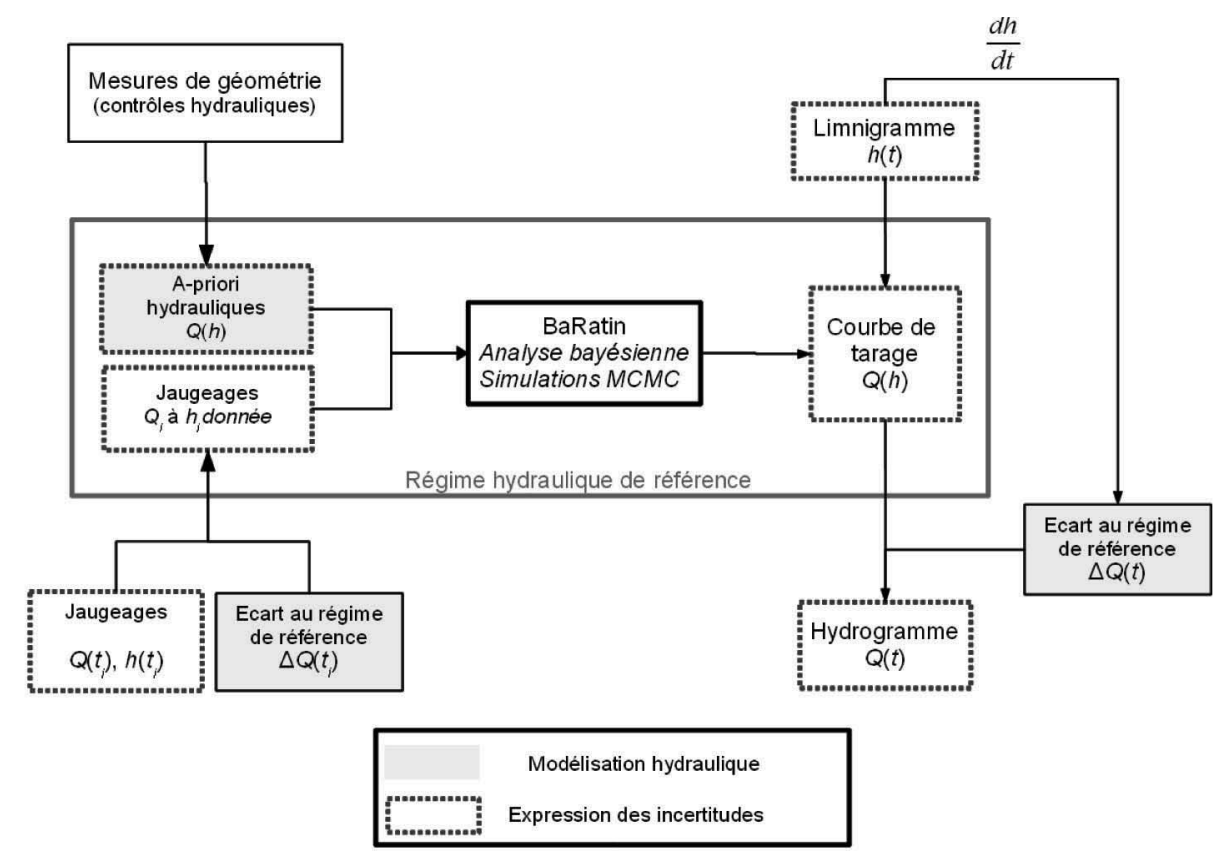

Figure 1 : Principes de la méthode BaRatin 
le contrôle par un chenal (cas des lits mineur et majeur). Dans le premier cas, la relation hauteur-débit est déterminée presque uniquement par la géométrie de la section de contrôle, où l'écoulement passe par le régime critique, ce qui se matérialise par une chute et une ligne d'eau quasi horizontale en amont. Dans le second cas, l'écoulement supposé uniforme est gouverné par la géométrie et la résistance à l'écoulement (" rugosité ») du chenal sur l'ensemble du tronçon homogène.

\section{II.1.2. Formalisation mathématique}

Des formules hydrauliques simples ${ }^{1}$ permettent de prédire de façon assez robuste la relation hauteur-débit liée aux différents contrôles hydrauliques les plus communs (cf. le guide pratique [Le Coz et al., 2011a], chapitre 10 pour plus de détails sur les formules d'ouvrages hydrauliques usuelles) :

- écoulement uniforme dans un chenal supposé de largeur constante $L$ et de pente $J$ (loi de Manning-Strickler simplifiée) : $Q=K L \sqrt{J}\left(h-h_{0}\right)^{5 / 3}$, avec $K$ le coefficient de Strickler de résistance à l'écoulement et $h_{0}$ le niveau moyen du fond du chenal ;

- seuil ou déversoir à crête horizontale de largeur $L$ et de cote $h_{0}: Q=C L \sqrt{2 g}\left(h-h_{0}\right)^{3 / 2}$, avec $g=9,81 \mathrm{~m} / \mathrm{s}^{2}$ l'accélération de la pesanteur et $\mathrm{C}=0,4$ pour un déversoir idéal ;

- seuil ou déversoir à crête triangulaire d'angle d'ouverture $\alpha$ et de cote au sommet $h_{0}: Q=\operatorname{Ctan}(\alpha / 2) \sqrt{2 g}\left(h-h_{0}\right)^{5 / 2}$, avec $\mathrm{C}=0,31$ pour un déversoir idéal ;

- orifice dénoyé de surface $S$ et de cote au centre $h_{0}$ : $Q=C S \sqrt{2 g}\left(h-h_{0}\right)^{1 / 2}$, avec C $=0,6$ pour un orifice complètement contracté

On remarque que l'ensemble de ces formules peuvent s'écrire en utilisant l'équation type suivante, à trois paramètres $a, b$ et $c$ :

$$
Q=a(h-b)^{c},
$$

Afin de pouvoir couvrir la plupart des configurations habituelles, dans lesquelles des contrôles se succèdent ou s'ajoutent, la courbe de tarage est mise en équation avec la formule mathématique générique suivante ${ }^{2}$ :

$$
Q=\sum_{r=1}^{N_{\text {segments }}} 1_{\left[\kappa_{r-1} ; \kappa_{r}\right]}(h) \sum_{j=1}^{N_{\text {controles }}} M(r, j) a_{j}\left(h-b_{j}\right)^{c_{j}},
$$

où $1_{\left[\kappa_{r-1} ; \kappa_{r}\right]}$ indique la fonction valant 1 sur l'intervalle de hauteur $\left[\kappa_{r-1} ; \kappa_{r}\right]$ définissant le segment d'indice $r$, et 0 en dehors de cet intervalle. La matrice $M$ est appelée matrice des contrôles hydrauliques : chacun de ses coefficients $M(r, j)$ vaut 1 si le contrôle hydraulique d'indice $j$, défini par une équation du type de l'Eq. (1), est actif sur le segment de hauteur d'indice $r$, et 0 sinon. Des conditions de continuité sont appliquées aux paramètres $b_{j}(j \geq 2)$ pour garantir le raccordement des différents tronçons de la courbe de tarage. L'utilisation pratique de cette équation et de la matrice des contrôles, rendue plus intuitive par l'interface graphique BaRatinAGE, sera illustrée plus en détail dans la Section III.

En vue de l'analyse BaRatin, la détermination des a priori hydrauliques va donc consister à identifier les contrôles hydrauliques en jeu pour la station étudiée, et à évaluer les hauteurs de transition et les valeurs des paramètres des formules hydrauliques associées. Il faut donc d'abord définir la structure de la courbe de tarage, à travers les hauteurs minimale et maximale d'exploitation de la station, $\kappa_{0}$ et $\kappa_{N_{s}}$ le nombre $N_{\text {segments }}$ de segments de courbe sur lesquels les contrôles hydrauliques sont homogènes, le nombre $N_{c o}$ de contrôles hydrauliques, leur type (chenal, ouvrage), et la façon dont ils se mettent en jeu en renseignant la matrice $M$ des contrôles hydrauliques. Ensuite, il faut spécifier la distribution a priori de chaque paramètre, à savoir les hauteurs de transition $\kappa_{r}$ pour $1 \leq r<N_{\text {segments }}$, et les paramètres $a_{j}$ et $c_{j}$ des formules hydrauliques. Seul le paramètre $b_{1}$, pour le

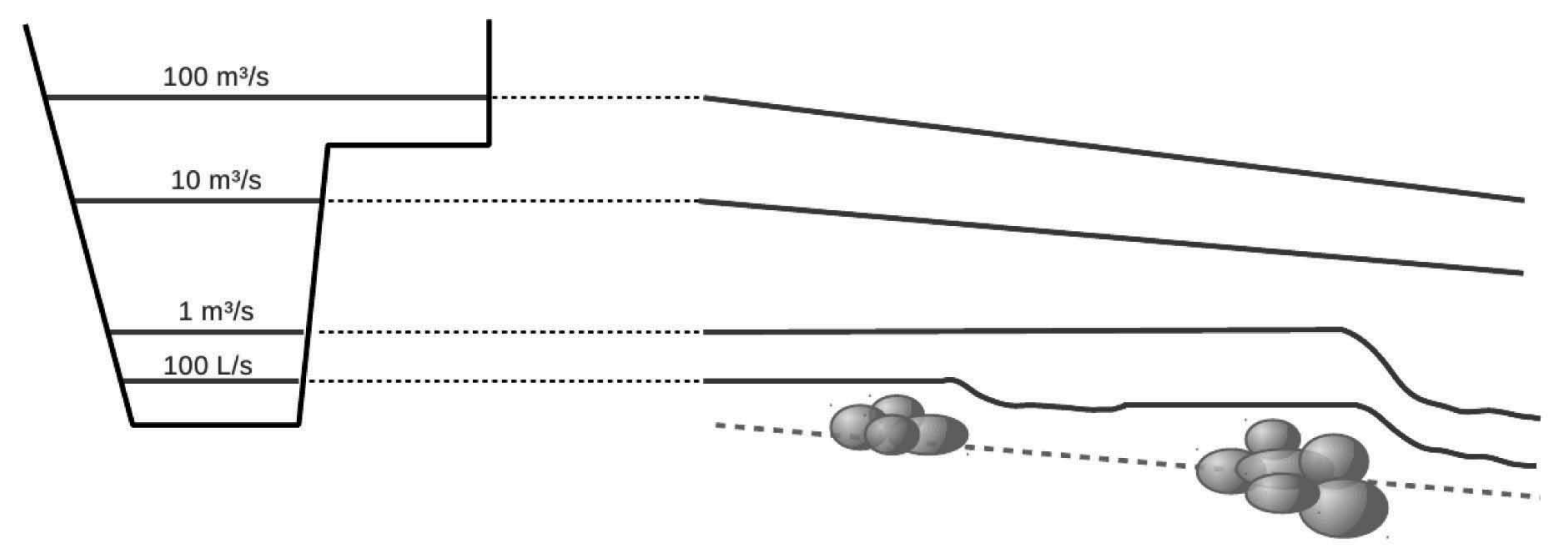

Figure 2 : Illustration simplifiée des contrôles hydrauliques pour une station hydrométrique typique : à droite, profil en long du fond et des lignes d'eau pour différentes valeurs de débit; à gauche, les niveaux d'eau sont reportés sur le profil en travers de la section de la station.

1. Dans ces formules, toutes les grandeurs doivent être exprimées dans les unités du Système International : longueurs en mètres, débits en $\mathrm{m}^{3} / \mathrm{s}$, etc.
2. Comme les contrôles sont supposés s'ajouter successivement, même quand leurs cotes d'activation sont similaires, il faut les distinguer. A noter que certains ouvrages, comme un déversoir triangulaire de largeur limitée composé avec un seuil horizontal, ne peuvent pas être représentés avec cette formulation. 
premier contrôle est à définir, les autres paramètres $b_{j}$ étant obtenus par continuité à partir des valeurs des paramètres $\kappa_{r}$.

En pratique, on définit la valeur moyenne de chaque paramètre et son incertitude, en spécifiant sa densité de probabilité. On suppose généralement une distribution gaussienne, définie par moyenne et écart-type, mais d'autres types de distribution (uniforme notamment) sont possibles. Les valeurs des exposants hydrauliques $c_{j}$ sont bien connues à partir des formules hydrauliques, donc les incertitudes a priori correspondantes sont très réduites. Cela a pour conséquence bénéfique de limiter l'interaction entre $a_{j}$ et $c_{j}$ et d'obtenir des valeurs de coefficients $a_{j}$ interprétables physiquement selon les formules hydrauliques auxquelles on se réfère.

\section{II.2. Critique et validation des jaugeages}

Dans l'inférence bayésienne réalisée par BaRatin (cf. Section II.3), l'information contenue dans les observations de la relation hauteur-débit (les jaugeages) va être confrontée aux a priori hydrauliques afin d'établir la courbe de tarage la plus probable et son faisceau d'incertitude, sous hypothèse stationnaire. Il convient donc de sélectionner l'ensemble des jaugeages disponibles, représentatifs de la relation hauteur-débit pour la période de stabilité considérée. Les détarages étant en général plus fréquents à bas débit, c'est pour cette gamme que la sélection des jaugeages est la plus importante. Une attention particulière doit être portée sur la qualité des mesures de débit, selon la technique employée et les métadonnées disponibles, mais aussi sur la fiabilité et la continuité de la référence des hauteurs lues. Il est courant lors des ré-analyses de stations que des erreurs soient décelées, sans toujours pouvoir être corrigées, dans les hauteurs jaugées à cause du remplacement, du déplacement ou d'une multiplicité d'échelles limnimétriques.

Une des originalités de la méthode BaRatin, par rapport aux méthodes bayésiennes publiées sur les courbes de tarage, est de permettre la prise en compte d'incertitudes différentes pour chaque jaugeage. C'est un avantage intéressant pour pouvoir utiliser des jaugeages plus incertains ou des estimations de débit dans des gammes peu ou pas jaugées, en pondérant leur contribution. Comme pour les paramètres des a priori hydrauliques, il est capital de spécifier des valeurs d'incertitudes réalistes, ni trop élevées ni trop faibles, aux jaugeages, car elles impactent à la fois le tracé de la courbe résultat et la largeur de son faisceau d'incertitude.

L'incertitude sur le débit jaugé, exprimé à un niveau de confiance de $95 \%$, peut être estimée à partir des informations disponibles sur les mesures de débit, ou calculée par des méthodes de propagation des incertitudes. Il reste difficile voire impossible d'appliquer des méthodes métrologiques classiques, à cause de la difficulté de mettre en équation les erreurs dues à l'environnement de mesure, souvent prépondérantes, et aussi à cause de l'absence de raccordement de la mesure de débit en rivière aux étalons internationaux. Des méthodes de calcul sont néanmoins en cours de développement, comme par exemple la méthode $\mathrm{Q}+[\mathrm{Le}$ Coz et al., 2012] pour les jaugeages par exploration des vitesses, en cours d'implémentation dans le logiciel BAREME [Bechon et al., 2013]. Les comparaisons inter-laboratoire permettent également de déterminer empiriquement des niveaux d'incertitude fiables sur des techniques hydrométriques comme le jaugeage par ADCP mobile par exemple.

En pratique, des valeurs typiques peuvent être affectées à chaque jaugeage selon la technique employée et des indicateurs de qualité. Le lecteur est invité à se reporter au guide pratique [Le Coz et al., 2011a] pour des valeurs classiques et des méthodes d'évaluation simples.

\section{II.3. Inférence bayésienne}

BaRatin est l'application du paradigme bayésien [Gelman et al., 2004] pour le problème de l'estimation des paramètres de la courbe de tarage, sur la base de jaugeages incertains et des connaissances hydrauliques.

\section{II.3.1. Modèle et hypothèses}

Le modèle statistique de BaRatin suppose que les erreurs sur les mesures de niveau sont négligeables ou sont reportées dans les incertitudes de débit, et que les mesures de débit sont affectées par des erreurs gaussiennes de moyenne nulle (pas de biais) et d'écart-type $u_{O}$, assimilée à l'incertitude-type sur les jaugeages évaluée précédemment (Section II.2). La courbe de tarage est formalisée comme une fonction $f(h \mid \boldsymbol{\theta})$, où $h$ est le niveau d'eau et $\boldsymbol{\theta}=\left(\theta_{1}, \ldots, \theta_{m}\right)$ sont les $m$ paramètres de la courbe de tarage. La fonction $f$ est donnée par l'Eq. 2 et les paramètres sont ceux listés à la fin de la Section II.1. On suppose que l'écart entre le débit réel et sa représentation mathématique $f$, forcément simplifiée, est une erreur gaussienne de moyenne nulle et d'écart-type $\sigma_{f}$, ce dernier étant inconnu et devant donc être estimé. On suppose également que cette erreur est indépendante de l'erreur de jaugeage.

On aboutit ainsi au modèle d'erreur suivant :

$\tilde{Q}_{i}=f\left(\tilde{H}_{i} \mid \boldsymbol{\theta}\right)+\varepsilon_{i}^{f}+\varepsilon_{i}^{Q} \operatorname{avec}: \varepsilon_{i}^{f}+\varepsilon_{i}^{Q} \sim N\left(0, \sqrt{\sigma_{f}^{2}+u_{Q_{i}}^{2}}\right)(3)$

où $\tilde{H}_{i}$ et $\tilde{Q}_{i}$ sont les hauteurs et débits jaugés, et $\varepsilon_{i}^{f}$ et $\varepsilon_{i}^{Q}$ sont les erreurs gaussiennes sur la formulation mathématique de la courbe de tarage et sur les débits jaugés, respectivement. L'Eq. (3) stipule donc que le débit jaugé est égale au débit prédit par la courbe de tarage, plus une erreur liée à l'incertitude de jaugeage, plus une erreur liée à l'imperfection de la courbe de tarage.

L'Eq. (3) comporte plusieurs quantités inconnues: les paramètres de la courbe de tarage $\boldsymbol{\theta}=\left(\theta_{1}, \ldots, \theta_{m}\right)$ et l'écart-type « restant $\sigma_{f}$. L'inférence sur ces quantités réclame de définir une vraisemblance et une distribution a priori comme décrit ci-après.

\section{II.3.2. Information portée par les données : vraisemblance}

La vraisemblance quantifie l'information portée par les jaugeages. De manière simplifiée, la vraisemblance correspond à la probabilité d'avoir observé les données, conditionnellement au modèle statistique (ici, l'équation 3) et à ses paramètres. On peut la calculer en remarquant que d'après l'équation (3), un débit jaugé $\tilde{Q}_{i}$ suit une loi normale de moyenne $f\left(H_{i} \mid \theta\right)$ (i.e. le débit prédit par la courbe de tarage) et d'écart-type $\sqrt{\sigma_{f}^{2}+u_{Q_{i}}^{2}}$. En supposant que chaque débit jaugé est indépendant, on obtient la vraisemblance suivante :

$$
p\left(\tilde{\boldsymbol{Q}} \mid \boldsymbol{\theta}, \sigma_{f}, \tilde{\boldsymbol{H}}\right)=\Pi_{i=1}^{N} \quad p_{G}\left(\tilde{Q}_{i} \mid f\left(\tilde{H}_{i} \mid \boldsymbol{\theta}\right), \sqrt{\sigma_{f}^{2}+u_{Q_{i}}^{2}}\right)(4)
$$

où $\tilde{\boldsymbol{Q}}=\left(\tilde{Q}_{1}, \ldots, \tilde{Q}_{N}\right)$ sont les $N$ débits jaugés et $p_{G}(z \mid m, s)$ représente la densité de probabilité d'une loi gaussienne de moyenne $m$ et d'écart-type $s$, évaluée en une valeur $z$. 


\section{II.3.3. Information liée aux connaissances hydraulique: distribution a priori}

La distribution a priori permet d'intégrer les connaissances hydrauliques discutées en section II.1. On supposera des a priori indépendants sur chaque paramètre à estimer, conduisant à :

$$
p\left(\boldsymbol{\theta}, \sigma_{f}\right)=p\left(\sigma_{f}\right) \prod_{i=1}^{m} p\left(\theta_{i}\right)
$$

La spécification des a priori a été discutée en section II.1, et sera illustrée dans le cas d'étude (section III).

\section{II.3.4. Théorème de Bayes et distribution a posteriori}

L'estimation des paramètres est effectuée à travers la distribution a posteriori, dont la fonction de densité de probabilité (pdf) est définie comme suit, selon le théorème de Bayes :

$$
\underset{\text { a posteriori }}{p\left(\boldsymbol{\theta}, \sigma_{f} \mid \tilde{\boldsymbol{H}}, \tilde{\boldsymbol{Q}}\right) \propto p} \underset{\text { vraisemblance }}{\left(\tilde{\boldsymbol{Q}} \mid \boldsymbol{\theta}, \sigma_{f}, \tilde{\boldsymbol{H}}\right) p\left(\boldsymbol{\theta}, \boldsymbol{\sigma}_{f}\right)}
$$

où le symbole $\propto$ signifie « est proportionnel à ». On notera que par rapport à des méthodes d'estimation plus classiques (ajustement d'une courbe de tarage par moindres carrés par exemple), le résultat de l'analyse bayésienne n'est pas seulement une valeur estimée des paramètres, mais plutôt une distribution (a posteriori) des paramètres. Ceci est un avantage en termes de quantification des incertitudes, puisque cette distribution représente directement l'incertitude d'estimation des paramètres de la courbe de tarage. Par contre, ceci induit une difficulté technique apparente : manipuler une distribution multivariée telle que (6) qui, de plus, n'est connue qu'à une constante de proportionnalité près, peut sembler rédhibitoire. Heureusement, cette difficulté peut être surmontée efficacement en utilisant des algorithmes de simulation MCMC.

\section{II.3.5. Utilisation de la distribution a posteriori : simulations $M C M C$}

Les algorithmes MCMC désignent une famille de méthodes qui permettent de simuler des réalisations à partir d'une densité de probabilité arbitraire, connue éventuellement seulement à une constante près. Une présentation détaillée des algorithmes MCMC en général, et de celui utilisé dans BaRatin en particulier, dépasse largement le cadre de cet article [cf. Renard et al., 2006, pour plus de détails]. On se contentera donc de mentionner que les simulation MCMC permettent de générer un grand nombre $M$ de réalisations $\left(\boldsymbol{\theta}^{(j)}, \boldsymbol{\sigma}_{f}^{(j)}\right)_{j=1: M}$ issues de la distribution a posteriori. A chacune de ces réalisations correspond une courbe de tarage (de paramètres $\boldsymbol{\theta}^{(j)}$ ), ce qui conduit à générer un ensemble de courbes de tarage plausibles au vu des jaugeages et des connaissance hydrauliques a priori. Typiquement, on réalise 100000 simulations de courbes de tarage pour les applications opérationnelles de BaRatin.

\section{APPLICATION OPÉRATIONNELLE}

\section{III.1. Interface graphique BaRatinAGE}

Afin de faciliter l'usage de BaRatin et de rendre possible son appropriation par le monde opérationnel, une interface graphique a été réalisée en langage tcl/tk (BaRatinAGE : BaRatin Advanced Graphical Environment). Elle permet d'entrer confortablement les résultats de l'analyse hydraulique et les valeurs et incertitudes a priori des paramètres de l'Eq. (2) avant de lancer BaRatin (cf. Figure 3). Dans la partie gauche, la configuration hydraulique du site (activation et désactivation des contrôles) se fait via un simple système de boutons cliquables et l'inscription des bornes des segments de hauteurs. Dans la partie droite, l'utilisateur renseigne les a priori qu'il a calculés pour chacun des contrôles hydrauliques identifiés.

En plus de la visualisation des résultats (courbe la plus probable et faisceau d'incertitude à un niveau de confiance de $95 \%$ ), cette interface propose un export vers le format hyd reconnu par BAREME, le logiciel de stockage et d'exploitation des jaugeages et courbes de tarage très largement utilisé par la communauté des hydromètres français. En attendant le couplage plus étroit en préparation entre

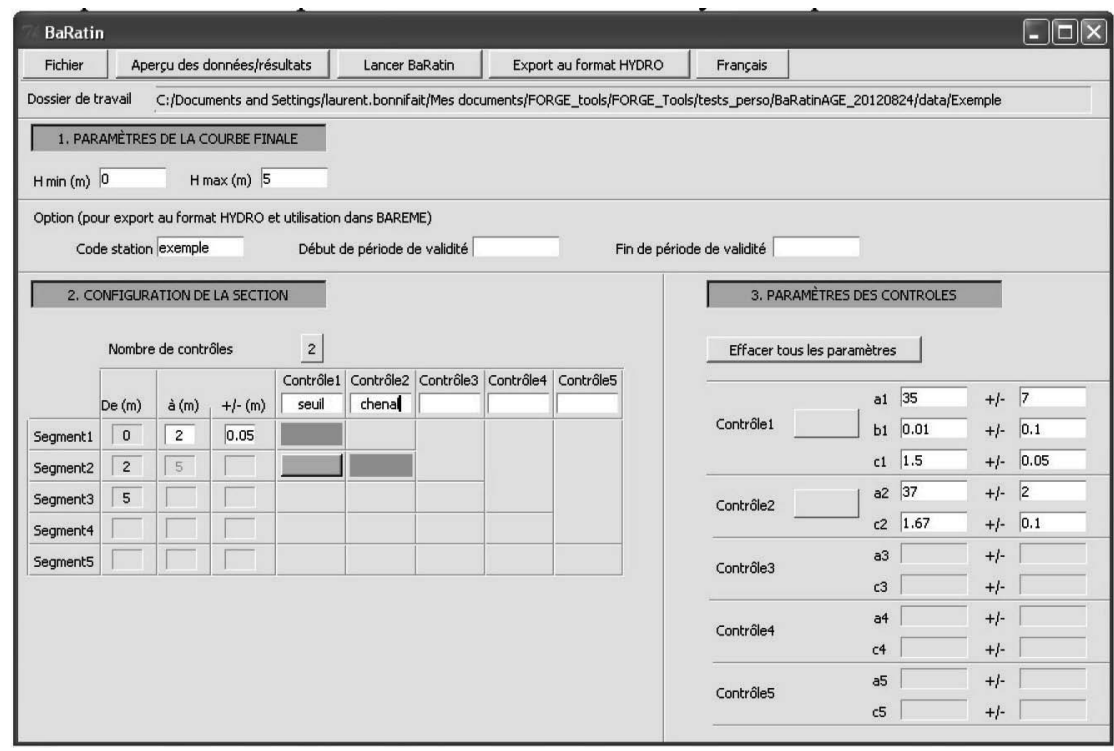

Figure 3 : Interface graphique de BaRatin 
BAREME et BaRatin, les jaugeages à exploiter par BaRatin sont à inscrire dans un fichier .xls qui permet de sélectionner ou désélectionner des jaugeages et d'affecter à chacun une incertitude appropriée.

Un fichier d'aide au calcul des valeurs et incertitudes a priori des paramètres $a, b, c$ des contrôles hydrauliques (voir formules Section II.1) est proposé également au format .xls. Ce format a l'avantage de pouvoir être exploité par les tableurs les plus populaires, qu'ils soient libres (Open Office, Libre Office) ou propriétaires (Excel). Si à terme les fonctionnalités de ces fichiers seront réparties entre les nouvelles versions de BAREME et BaRatinAGE, l'utilisation d'un format très répandu a pour enjeu qu'en attendant, leur utilisation ne pose pas de difficultés aux utilisateurs.

Que ce soit pour les jaugeages comme pour les a priori hydrauliques et les résultats, dans l'interface BaRatinAGE les incertitudes correspondent toujours à un niveau de confiance de $95 \%$. Dans les cases précédées du symbole \pm , la valeur du demi-intervalle doit être renseignée.

\section{III.2. Exemple d'application à une station hydrométrique}

\section{III.2.1. Analyse du fonctionnement hydraulique du site}

On prend ici comme exemple opérationnel l'analyse de la station de la Sarre à Sarrebourg (code Hydro A9021010, bassin versant de $311 \mathrm{~km}^{2}$ ) réalisée par [Le Boursicaud et al., 2012] pour le SPC Rhin-Sarre. La station de la Sarre à Sarrebourg a été installée en 1953 à l'amont immédiat d'un pont SNCF d'environ $50 \mathrm{~m}$ de long et comprenant trois arches et un tunnel routier (Figure 4). La présence d'un large remblai en rive gauche provoque un important rétrécissement du lit également visible sur la Figure 4. En cas de submersion du remblai, l'élargissement de la section a un important effet sur l'hydraulique du tronçon.

Après analyse du site, son fonctionnement hydraulique peut se décomposer en trois gammes de hauteur correspondant à des contrôles hydrauliques homogènes :

- Gamme 1 : Le contrôle hydraulique, peu sensible et potentiellement instable, est ici assuré par un radier naturel de type gravelo-sableux, situé sous l'arche de droite.
Ce contrôle pourra être représenté par une formule de seuil à crête horizontale.

- Gamme 2 : Le contrôle dépend de la géométrie et de la rugosité du chenal situé au pied du remblai. Ce fonctionnement pourra se modéliser par une relation de Manning-Strickler simplifiée.

— Gamme 3 : Le fonctionnement hydraulique est contrôlé à la fois par le lit mineur et le lit moyen (remblai submergé). Ainsi nous utilisons deux relations de Manning-Strickler pour cette gamme : celle du lit mineur, à laquelle vient s'ajouter celle du lit moyen situé au dessus du banc.

Une modélisation hydraulique a permis de consolider cette analyse des contrôles hydrauliques et de préciser les hauteurs de transition entre gammes. Un modèle 1D (logiciel MAGE, Irstea) a été construit à partir de 9 profils en travers, avec une description raffinée autour de la station et du pont, et une extension aval suffisante pour s'assurer de la dissipation de la condition aval. Cette modélisation confirme notamment que les orifices du pont SNCF, à savoir les trois arches et le tunnel routier, ne semblent pas opérer de contrôle significatif avant leur mise en charge, qui correspond à la limite supérieure d'exploitation de la station. Au-delà de cette limite (5 m à l'échelle), le pont SNCF est contourné au dessus des voies ferrées en rive droite, l'abri de la station est atteint et le capteur est vraisemblablement hors d'état de marche. Le modèle montre en revanche l'important impact hydraulique du remblai, qui masque l'arche gauche du pont SNCF et induit une nette inflexion dans la relation hauteur-débit lorsqu'il est submergé. Cet effet est bien plus sensible que la mise en eau du tunnel routier, qui intervient vers $3 \mathrm{~m}$ à l'échelle.

\section{III.2.2. Paramétrage des a priori hydrauliques}

Les différentes gammes de contrôles et leur paramétrage a priori fourni en entrée de BaRatin sont résumés sur les Figures $5 \mathrm{a}$ et $5 \mathrm{~b}$. Les valeurs centrales des paramètres $a, b$, $c$ et leur incertitude ont été estimées simplement, soit à partir de la topographie et des formules hydrauliques usuelles (cas de la gamme 1), soit par calage sur les résultats de la modélisation 1D (cas des gammes 2 et 3 ). Dans ce cas, une courbe de tarage centrale et deux courbes enveloppes ont été calculées en considérant des coefficients de Strickler réalistes, sous hypothèse de rugosité moyenne, faible et forte.

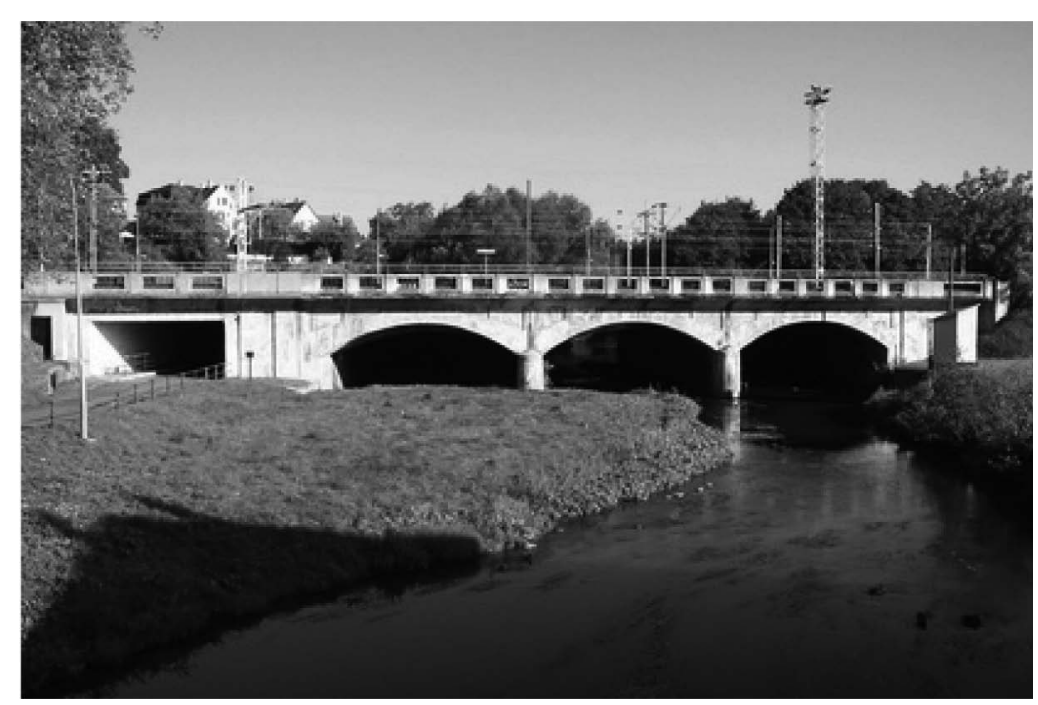

Figure 4 : Vue, à bas débit et depuis l'amont, de la station de la Sarre à Sarrebourg (code Hydro A9021010) avec au fond le pont SNCF situé en aval et au premier plan le remblai submersible situé en rive gauche 
La hauteur de transition entre les gammes 2 et 3 a été fixée à la cote moyenne du remblai en rive gauche, à partir des données de topographie disponibles. La transition entre les gammes 1 et 2 correspond à l'ennoiement du radier naturel. En l'absence de chute prononcée, elle a été fixée arbitrairement à $20 \mathrm{~cm}$ au-dessus de la cote supposée du radier, en laissant une incertitude a priori assez large.

\section{III.2.3. Sélection et critique des jaugeages}

Les jaugeages disponibles de 1995 à aujourd'hui s'étendent de $1 \mathrm{~m}$ à $3 \mathrm{~m}$ à l'échelle, avec une fréquence moyenne d'environ 6 jaugeages par an. Les techniques employées sont l'exploration du champ des vitesses, à pied et par camion hydrométrique, et le profileur hydro-acoustique (ADCP) pour les mesures les plus récentes, notamment pour les deux plus forts débits jaugés alors que le remblai était submergé. Par simplification, une incertitude de $\pm 7 \%$ a été supposée pour tous les jaugeages (Figure 5d). Dans le cas de jaugeages de crue dégradés, par exemple réalisés par des méthodes non intrusives (flotteurs, vidéo, radar), une incertitude plus forte aurait pu être prise en compte pour ne pas biaiser l'évaluation du haut de la courbe.

Les données de jaugeage montrent une importante dispersion à bas débit, mais aucune période ou saison de stabilité n'a pu être identifiée facilement. Tous les jaugeages à bas débit ont été conservés, en considérant en première approche que la dispersion des jaugeages est représentative de l'incertitude sur le bas de la courbe, en raison de l'instabilité et du manque de sensibilité de la station. Un seul jaugeage a été écarté de l'étude, après identification

\begin{tabular}{|c|c|c|c|c|c|c|}
\hline & \multicolumn{3}{|c|}{ Nombre de contrôles } & \multicolumn{3}{|l|}{3} \\
\hline & \multirow[b]{2}{*}{$\mathrm{De}(\mathrm{m})$} & \multirow[b]{2}{*}{ à (m) } & \multirow[b]{2}{*}{$+f-(\mathrm{m})$} & Contrôle1 & Contrôle2 & Contrôle3 \\
\hline & & & & radier & mineur & banc \\
\hline Segment1 & 1 & 1.2 & 0.3 & & & \\
\hline Segment2 & 1.2 & 2.6 & 0.5 & & & \\
\hline Segment3 & 2.6 & 3.8 & & & & \\
\hline
\end{tabular}

(a)

\begin{tabular}{|c|c|c|c|c|}
\hline \multirow{3}{*}{ Contrôle 1 } & & 14 & $+1-$ & 3 \\
\hline & b1 & .95 & $+1-$ & .25 \\
\hline & $c 1$ & 1.5 & $+1-$ & 0.05 \\
\hline \multirow{2}{*}{ Contrôle2 } & a2 & 8 & $+1-$ & 2 \\
\hline & $c 2$ & 1.66 & $+1-$ & 0.05 \\
\hline \multirow{2}{*}{ Contrôle3 } & a3 & 80 & $+1-$ & 20 \\
\hline & $c 3$ & 1.66 & +1 & 0.05 \\
\hline
\end{tabular}

(b)

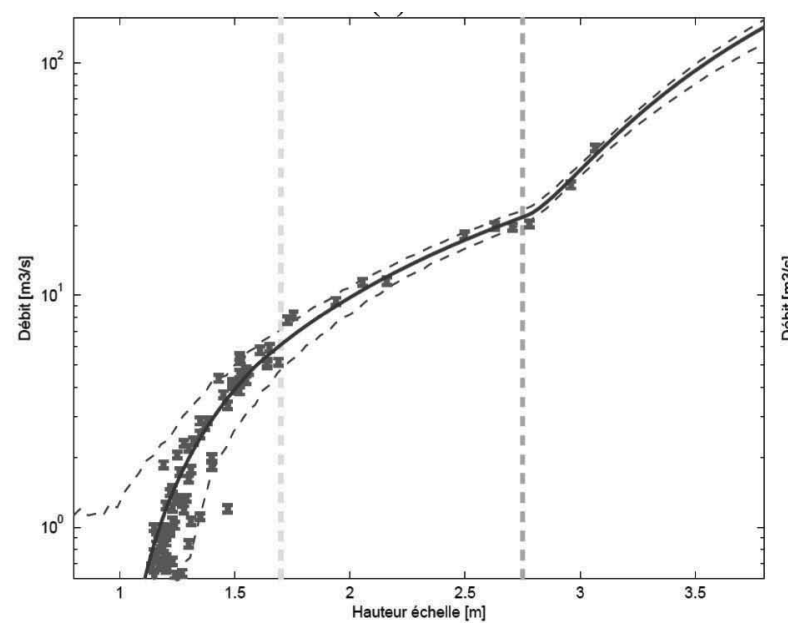

(d)

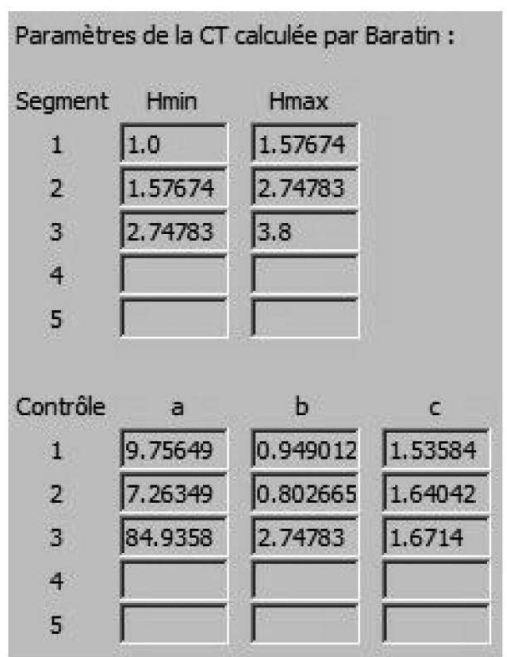

(c)

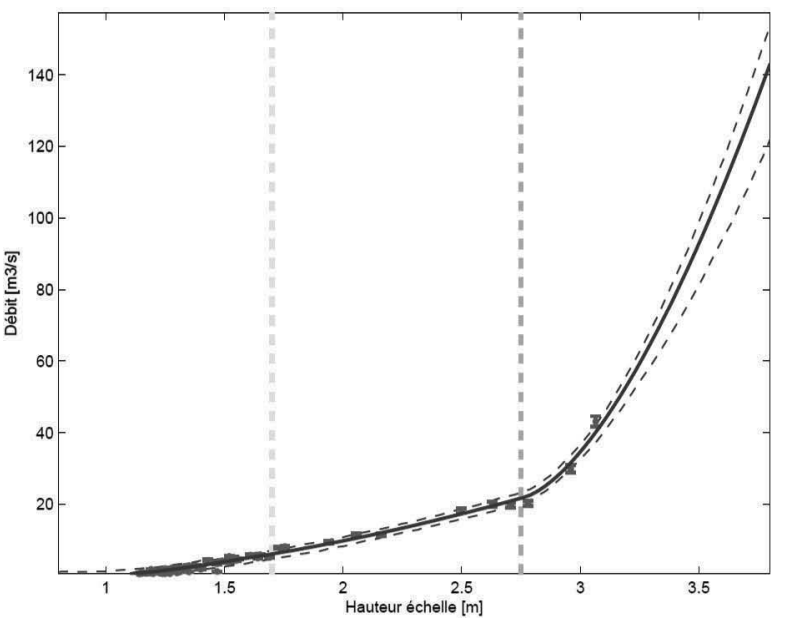

(e)

Figure 5 : Application de la méthode BaRatin à la station de la Sarre à Sarrebourg : contrôles hydrauliques identifiés a priori (a) ; paramètres des 3 contrôles hydrauliques identifiés : valeurs centrales et incertitudes a priori (b), à comparer aux valeurs calculées par BaRatin (c); courbe de tarage calculée par Baratin, avec l'enveloppe d'incertitude à 95\% et les jaugeages assortis de leur incertitude, ici $\pm 7 \%$ pour tous : avec les débits en échelle linéaire (d) et en échelle logarithmique (e). Les hauteurs de transition entre gammes de contrôle sont indiquées par des lignes verticales pointillées (jaune et cyan). 
d'un problème lié à la technique de mesure employée à l'époque.

\section{III.2.4. Résultats de l'analyse BaRatin}

Les résultats numériques de l'analyse BaRatin sont présentés sur les Figures $5 \mathrm{c}$ et $5 \mathrm{~d}$. Dans l'ensemble les paramètres calculés par BaRatin s'écartent peu des a priori fournis en entrée. La hauteur de transition entre les gammes 1 et 2 , estimée arbitrairement a priori à $1,2 \mathrm{~m}$, est ré-évaluée à $1,6 \mathrm{~m}$, ce qui reste tout à fait réaliste. Les jaugeages disponibles entre $2,50 \mathrm{~m}$ et $3 \mathrm{~m}$ permettent de préciser la hauteur de transition entre les gammes 2 et 3 , donnée à $2,75 \mathrm{~m}$. La largeur du radier de basses eaux est revue à la baisse, à une valeur réaliste également : $5,5 \mathrm{~m}$ en résultat $\left(a_{1}=9,7\right)$ au lieu de $8 \mathrm{~m}$ a priori $\left(a_{1}=14\right)$.

L'affichage graphique des résultats de BaRatin (Figure 5d) montre un intervalle d'incertitude relativement réduit, sauf pour les bas débits, en raison de la dispersion des jaugeages. Rappelons toutefois que l'incertitude fournie ne porte que sur la courbe de tarage (comme estimation de la relation hauteur-débit), et non pas sur l'hydrogramme qui en est issu. A l'exception d'un jaugeage en étiage, toutes les barres d'erreur des jaugeages intersectent l'intervalle d'incertitude à $95 \%$, bien que celui-ci soit assez resserré.

Les deux jaugeages les plus élevés témoignent de l'inflexion brutale de la relation hauteur-débit due à la submersion du remblai, qui est respectée par la courbe BaRatin et conforme aux a priori. Ces deux jaugeages de crue ont été réalisés en 2011 par mesure ADCP et filmés, ce qui a permis d'en confirmer la validité. En plus de la modélisation hydraulique, ils permettent de réduire sensiblement l'incertitude sur la gamme haute de la courbe de tarage.

\section{III.3. Appropriation par un service opérationnel}

\section{III.3.1. Application à la gestion des courbes de tarage}

La DRIEE Ile-de-France, comme tous les services d'hydrométrie, fait régulièrement évoluer ses courbes de tarage au fur et à mesure des modifications des conditions d'écoulement ainsi que de l'obtention de nouveaux jaugeages. Elle réévalue les extrapolations en hautes eaux de ses courbes de tarage en utilisant la méthode BaRatin qui constitue une méthode assez économe en données topographiques et en calculs lourds par rapport à une modélisation hydraulique par exemple.

La méthode est actuellement en cours de déploiement mais devrait à terme être utilisée systématiquement lors de la mise à jour ou de la création de courbes de tarages. Elle est utilisée prioritairement sur :

- les stations pour lesquelles on possède peu de jaugeages représentatifs des hautes eaux, ce qui correspond à des stations récentes ou bien pour lesquelles les configurations hydrauliques ont récemment changé, ainsi qu'aux rivières à réaction très rapide et plus généralement à toutes les stations pour lesquelles il est difficile de réaliser un bon jaugeage en crue ;

- les stations pour lesquelles les débordements en lit majeur sont importants, ce qui se traduit par un accroissement significatif de la débitance et donc de la pente de la courbe de tarage.

Le premier cas de figure est illustré par la station d'Epinay sur l'Orge ( $632 \mathrm{~km}^{2}$, dans le département de l'Essonne) soumis à l'influence d'un ouvrage hydraulique (environ $1 \mathrm{~km}$ à l'aval) abaissé en 2011 pour restaurer la continuité écologique de la rivière. C'est par ailleurs une station pour laquelle il est difficile de jauger en hautes eaux en raison de la rapidité de la réaction aux précipitations. Une analyse préliminaire des courbes de tarage avant et après abaissement du seuil a été conduite avec BaRatin, sur la base d'a priori hydrauliques relativement peu contraints, reproduisant notre connaissance fruste de la géométrie des contrôles à bas débit (seuil abaissé d'environ $40 \mathrm{~cm}$ ) et à débit intermédiaire (chenal supposé inchangé).

Les résultats sont présentés sur la Figure 6. A bas débit (hauteur inférieure à $1,50 \mathrm{~m}$ environ), les jaugeages contraignent suffisamment les courbes, en accord acceptable avec les a priori. Par contre, en contrôle par le chenal, le résultat est sensible aux quelques jaugeages disponibles au-dessus de 1,20 m échelle, qui sont assez dispersés (ces jaugeages seraient à étudier et re-critiquer plus finement). Des simulations BaRatin ont été conduites avec les deux jeux de jaugeages strictement séparés pour les deux périodes avant/après, et aussi en étudiant la sensibilité aux derniers jaugeages (d'avril à juin 2013 avec une crue jaugée en juin). On constate dans le premier cas que les courbes correspondantes divergent significativement en hautes eaux, avec certainement un fort poids pour le jaugeage élevé de juin 2013 (hauteur $1,95 \mathrm{~m}$ ). Noter que comme attendu, en hautes eaux la courbe en vigueur (corrigée grâce aux jaugeages récents du printemps 2013) est proche de la courbe calculée. Ce cas illustre bien à la fois l'intérêt d'une modélisation hydraulique du tronçon (prévue pour fin 2013) et la nécessité de s'organiser pour réaliser les jaugeages des crues importantes. Préciser le haut extrapolé des courbes de tarage avec des observations plutôt que des calculs nécessairement simplifiés nous apparaît une démarche bien plus robuste, pourvu que les mesures soient suffisamment précises et documentées.

Le second cas est illustré par la station de Gy sur la rivière Ouanne $\left(883 \mathrm{~km}^{2}\right.$, dans le département du Loiret). La station est située à l'aval immédiat d'un pont et d'une route remblayée. Les débordements en lit majeur droit sont importants mais assez bien connus car jaugés à plusieurs reprises. Le lit majeur, rectangle de cote $1,5 \mathrm{~m}$ environ et de largeur moyenne $350 \mathrm{~m}$, est occupé par une peupleraie et des hautes herbes. La configuration apparaît stable depuis le milieu des années 1990. Le lit mineur est large de $30 \mathrm{~m}$ environ, avec un radier de basses eaux plus étroit. Avec ces a priori et des hypothèses sur la pente moyenne et la résistance à l'écoulement de chaque lit, une analyse BaRatin a été menée pour les jaugeages en vigueur (Fig. 7).

Les résultats fournis par BaRatin sont suffisamment bien contraints par les jaugeages disponibles, et conformes à notre connaissance a priori des contrôles hydrauliques, et d'ailleurs proches de la courbe de tarage en vigueur. Ceci permet de conforter sur une base physique l'extrapolation en hautes eaux non jaugées restant contenues dans le lit majeur. Par la suite, BaRatin sera testé comme outil pour optimiser le nombre de courbes de tarage (la chronique de 37 années de la station de Gy étant décrite par 12 courbes de tarage successives) via des tests sur la durée des jeux de données et la sensibilité à l'incertitude sur les jaugeages.

\section{III.3.2. Intérêt pratique de la méthode}

La méthode associe des calculs scientifiques rigoureux et des raisonnements hydrauliques qui constituent en fait un équivalent numérique de l'interprétation graphique que pratique l'hydromètre connaissant bien les conditions d'écoulements de son site. BaRatin est très sensible à une bonne sélection et critique des mesures avec évaluation de leurs 


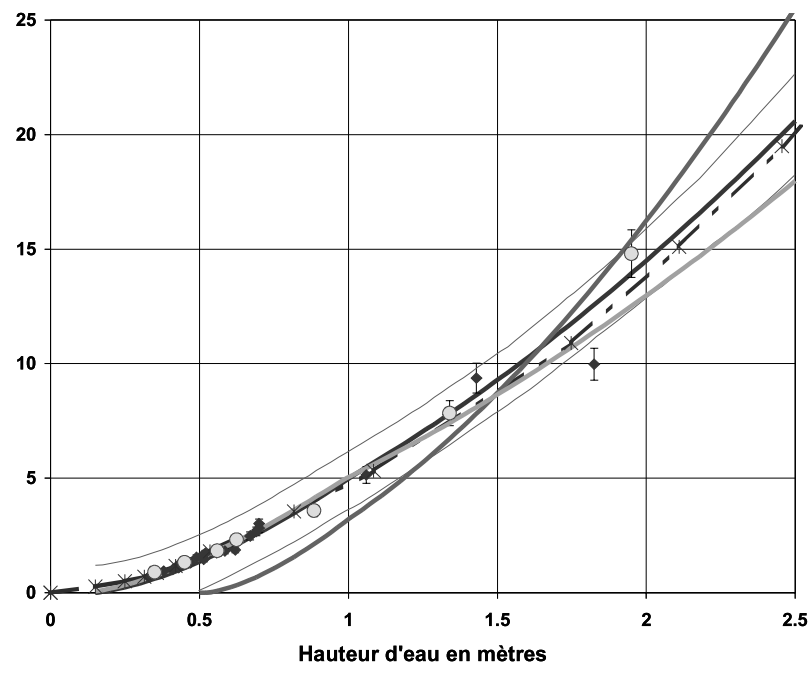

(a)

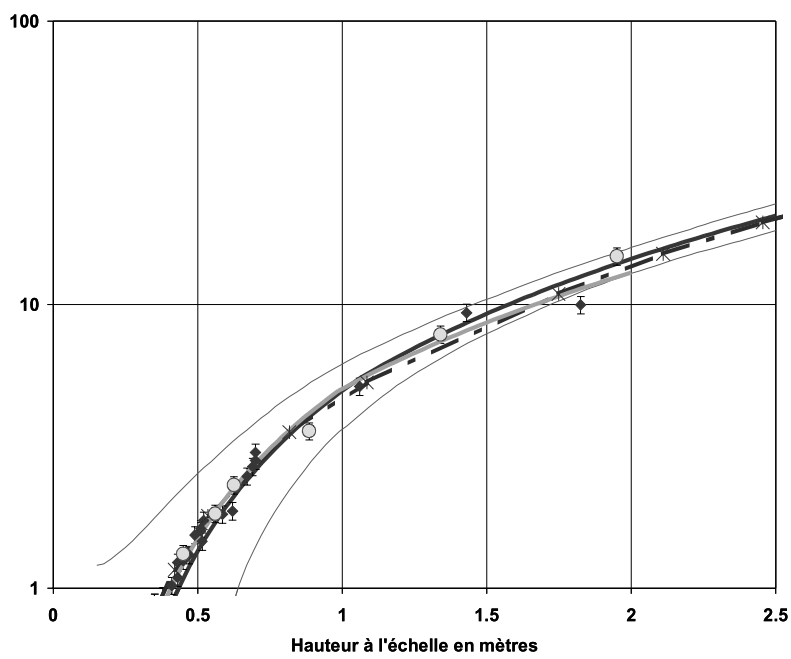

(b)

Figure 6 : Analyse préliminaire avec BaRatin de la station d'Epinay sur l'Orge : jaugeages assortis de leur incertitude ( $\pm 7 \%)$ et courbes de tarage les plus probables (MaxPost) avec les débits en échelle linéaire (a) et en échelle logarithmique (b). On distingue la situation actuelle (en bleu) et la situation avant l'abaissement définitif du seuil influençant la station (en rouge). La courbe pointillée violette représente la courbe réalisée par l'hydromètre indépendamment de BaRatin à l'été 2013. La courbe verte correspond à la courbe de tarage actuelle, calculée par BaRatin sans les jaugeages les plus récents (points jaunes).

incertitudes ; cela renforce notre démarche qui consiste notamment à recentrer notre analyse sur les mesures (après une analyse critique fine). Ceci rejoint les travaux menés maintenant depuis plusieurs années au sein de la DIREN IDF puis de la DRIEE pour améliorer la connaissance des débits de la crue de 1910 sur notre territoire [Lacaze et al,
2013]. C'est également très pédagogique pour illustrer l'influence des jaugeages en crue et montrer combien cela est crucial malgré toutes les difficultés engendrées sur le terrain et en terme d'organisation [Chaléon et al, 2013].

L'intérêt de la méthode pour les étiages est moins évident au sein de la DRIEE dans la mesure où les ajustements fins

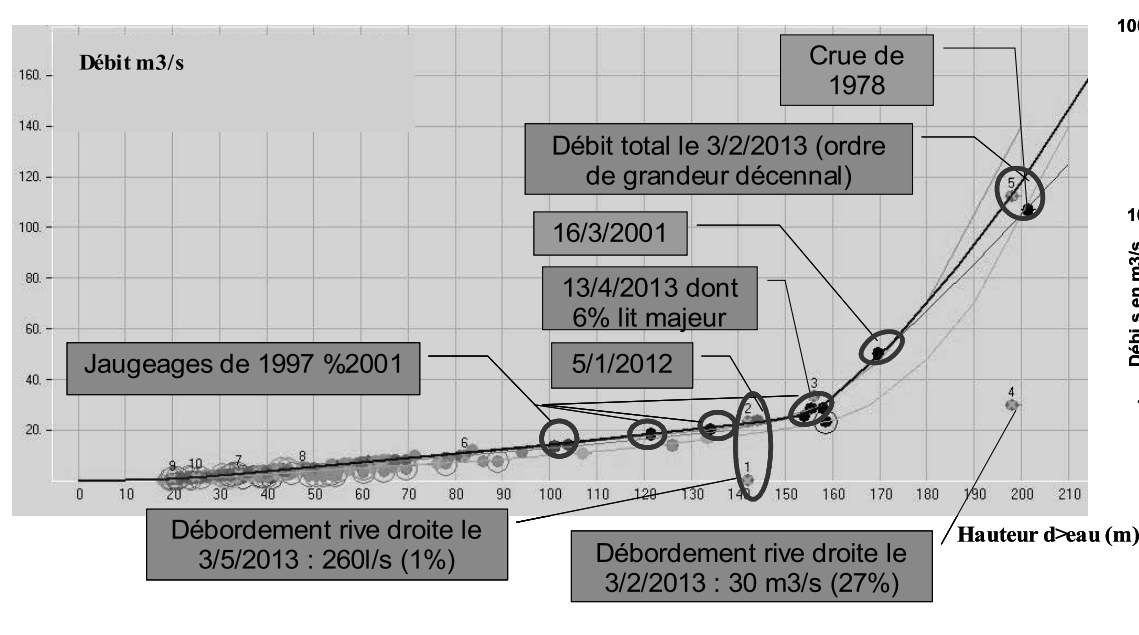

(a)

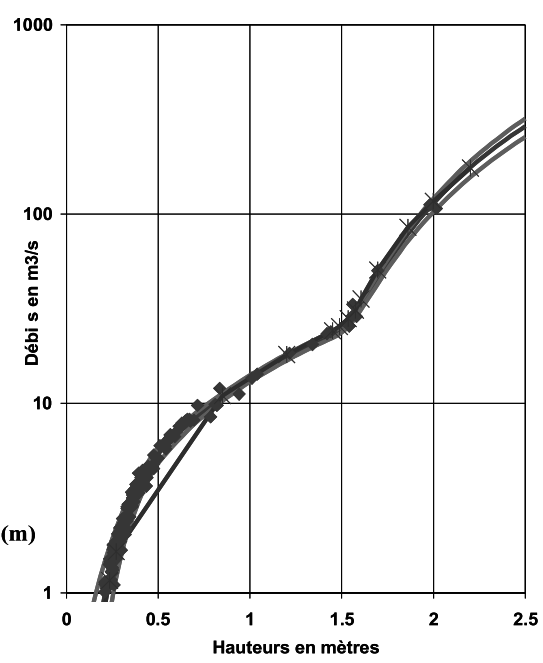

(b)

Figure 7 :. Analyse BaRatin de la courbe de tarage actuelle à la station de Gy sur l'Ouanne : (a) courbes de tarage établies avec le logiciel Barême (établies indépendamment de l'analyse BaRatin) où les jaugeages des débordements en lit majeur sont mis en évidence et les jaugeages anciens intéressants figurent en noir et (b) comparaison de la courbe de tarage en vigueur (violet) avec la courbe de tarage la plus probable (MaxPost, en bleu), les jaugeages assortis de leur incertitude ( $\pm 7 \%)$ selon une échelle logarithmique (b). Les hauteurs de transition entre gammes de contrôle sont indiquées par des lignes verticales pointillées (jaune et cyan). Le débordement en lit majeur est très marqué à partir de 1,50 $\mathrm{m}$ à l'échelle. Le graphique log permet de constater que la courbe de tarage en vigueur manque de résolution à bas débit. 
sont gérés mensuellement ( 2 fois par mois pour les stations caractéristiques de l'étiage) via la procédure CORTH de la Banque Hydro [Lacaze et al, 2009]. Cette procédure revient à créer des hauteurs fictives servant au calcul du débit via la courbe de tarage stable. Cela permet à la fois de conserver en base les hauteurs mesurées en rivière, de corriger finement les calculs de débit; les courbes de tarage ont, sauf exception, une validité de plusieurs années de manière à traduire les conditions hydrauliques intrinsèques pérennes de la section. Une révision de la courbe de tarage ou la création d'une nouvelle ne se justifie alors que si les caractéristiques de la section ou de son contrôle hydraulique aval sont durablement modifiées. Une perspective de développement de BaRatin est de pouvoir gérer des courbes de tarage nonstationnaires avec des paramètres variables dans le temps, dans les cas où la relation hauteur-débit subit des variations temporaires ou cycliques, sous l'effet de la végétation aquatique notamment. Cela permettrait de gérer de façon plus physique les détarages progressifs, et non pas indirectement à travers une correction fictive des niveaux mesurés.

\section{III.3.3. Rétro-activité des courbes de tarage et réanalyse des débits}

L'analyse par un service opérationnel de ses courbes de tarage avec la méthode BaRatin peut amener à des résultats suffisamment différents des barèmes antérieurs pour poser la question de les appliquer rétroactivement aux hydrogrammes passés. La validité des courbes proposées ne s'applique qu'à la période de stabilité des contrôles hydrauliques considérés dans l'étude. Dans l'exemple d'application à la station de la Sarre à Sarrebourg, on a vu que le fonctionnement hydraulique actuel est fortement influencé par le remblai situé en rive gauche à l'amont de la station. Ainsi pour connaître la période de validité dans le passé de la courbe proposée, il conviendra de remonter à la date d'apparition de ce remblai sous sa forme actuelle.

Le plus souvent, il faut alors distinguer la situation de la partie bien jaugée de la courbe, à bas et moyen débit, de celle de la partie hautes-eaux, souvent peu ou pas jaugée. Dans le premier cas, la courbe BaRatin est souvent très proche de la courbe antérieurement calée sur les mêmes jaugeages. Comme c'est à bas débit que les détarages marqués sont les plus fréquents, il est alors préférable de conserver les courbes de tarage existantes pour les différentes périodes de stabilité. En revanche en hautes eaux, la courbe BaRatin peut s'écarter sensiblement de l'extrapolation antérieure, sur la base d'un a priori hydraulique, appuyé sur une analyse topographique et idéalement d'une simulation numérique, qui n'était en général pas explicité auparavant. Sauf en cas de changement morphologique majeur dans le tronçon sur lequel est installé la station, la relation hauteur-débit est stable pour les forts débits. Il est alors cette fois préférable d'appliquer la partie haute de la courbe BaRatin à différentes périodes du passé.

En pratique, il est possible d'importer la courbe BaRatin dans le logiciel BAREME et de la superposer aux anciennes courbes afin de réaligner manuellement leur partie haute sur la courbe BaRatin, en ménageant une transition douce entre les deux parties de courbe. Si ces courbes de tarage réactualisées sont validées, elles peuvent alors être intégrées à la Banque Hydro. Cela se traduit par un recalcul automatique des chroniques de débit et de nouvelles valeurs des crues de référence (il semble que cela se fasse en quelques heures). L'impact est évidemment non négligeable pour les études hydrologiques, les PPRI etc. Aussi, si on ne veut pas modifier tout de suite les chroniques publiées (et les quantiles de crue affichés), il est possible de créer une station fictive sur le même bassin (et si besoin de la supprimer ensuite). En revanche, si on les modifie directement, mieux vaut insérer un avertissement en commentaire sur la page de présentation de la station, indiquant que l'hydrologie des crues au-dessus d'un débit donné a été réactualisée pour cette station.

\section{CONCLUSIONS ET PERSPECTIVES}

La méthode BaRatin a été appliquée à l'analyse de la courbe de tarage de stations hydrométriques variées, en termes de dimensions, de nature des contrôles hydrauliques, et de densité et incertitude des jaugeages disponibles (voir [Tolsa et al., 2013] et les rapports d'étude : [Bonnifait et al., 2011], [Le Coz et al., 2011b], [Le Boursicaud et al., 2012], [Le Coz, 2012]). Elle a permis d'obtenir des résultats intéressants sur des sites plus ou moins stables et densément jaugés, des sites avec contrôle hydraulique par ouvrage artificiel ou non, des sites avec débordement jaugé ou extrapolé, etc.

La méthode permet notamment d'évaluer le gain en termes d'incertitude apporté par l'effort de jaugeage, la présence d'un contrôle artificiel (seuil/déversoir), la connaissance ou la modélisation des contrôles hydrauliques, etc. La méthode permet aussi de valoriser des jaugeages à fort débit plus incertains, par flotteurs, radar ou analyse de séquences d'images, voire des estimations de débit de pointe par retour d'expérience.

Le logiciel BaRatin et son interface BaRatinAGE sont diffusés librement et gratuitement sous licence Irstea. Un couplage de BaRatin avec le module " Courbe de tarage » du logiciel BAREME est en cours de réalisation [Bechon et al., 2013]. Une perspective de développement envisagée pour cette méthodologie est la prise en compte de non-stationnarités graduelles (végétation) ou soudaines (crue) à l'aide de paramètres variables dans le temps. La méthode peut également s'appliquer à d'autres courbes d'étalonnage utilisées en hydrométrie, comme les courbes de tarage à deux niveaux, les relations vitesse moyenne / vitesse index, ou les relations turbidité / concentration, par exemple.

\section{REMERCIEMENTS}

BaRatin utilise la bibliothèque Fortran DMSL développée par Dmitri Kavetski de l'Université d'Adélaïde, en Australie. Le développement de la méthode a été soutenu par Irstea, le SCHAPI, l'OMM, l'ANR (projet FloodScale sur la modélisation multi-échelle des crues cévenoles) et, via un partenariat $\mathrm{PHC}$, par le ministère des Affaires étrangères et européennes et le ministère de l'Enseignement supérieur et de la Recherche. Le projet FloodScale est soutenu par l'Agence Nationale de la Recherche (ANR) contrat $n^{\circ}$ ANR 2011 BS56 027 qui contribue au programme HyMeX. La méthode a bénéficié des échanges avec les hydromètres et hydrologues des services opérationnels dont les stations ont été analysées ou sont en cours d'analyse, en particulier la DREAL Alsace et le SPC Rhin-Sarre. L'intégration dans le logiciel BAREME est réalisée par Pierre-Marie Bechon (DREAL Rhône-Alpes).

\section{REFERENCES}

Bechon P.-M., Le Coz J., Leleu I., Renard B. (2013) - Des outils du réseau Etat en hydrométrie et leur ouverture aux 
notions d'incertitude. Congrès SHF : «Hydrométrie 2013», Paris, 15-16 mai 2013

Branger F., Le Coz J., Renard B., Bonnifait L. (2012) Méthode d'estimation des incertitudes sur les courbes de tarage. Fiche technique OTHU, (disponible sur internet: www. graie.org/othu/pdfothu/fiches/F26-tarage.pdf). 4

Bonnifait L., Le Coz J., Renard B. (2011) - Analyse des courbes de tarage de 7 stations hydrométriques de la DREAL Alsace. $170 \mathrm{p}$

Chaleon C., Diribarne J., Marteau A., Marlette S., Garro J., VALENTE M. (2013) - Fiabilisation et diminution des incertitudes hydrométriques à la DRIEE Ile de France. Congrès SHF : «Hydrométrie 2013», Paris, 15-16 mai 2013

Gelman A., Carlin J.B., Stern H.S., Rubin D.B. (2004) Bayesian data analysis. Chapman \& Hall, 2nd Edition, Texts in Statistical Science

Iso/Ts 25377. (2007) - Hydrometric Uncertainty Guidance (HUG), ISO, Geneva (Switzerland)

Lacaze Y., Chesneau S., Chaleon C. (2013) - Analyse de cohérence des informations hydrométriques pour la validation du débit de la crue de 1910 à Paris. Congrès SHF : «Hydrométrie 2013», Paris, 15-16 mai 2013

Le Boursicaud R., Bonnifait L., Le Coz J., Renard B. (2012) Analyse des courbes de tarage de 5 stations hydrométriques pour le SPC Rhin-Sarre, rapport technique. $168 \mathrm{p}$

Le Coz J., Camenen B., Peyrard X., Dramais G. (2012) Uncertainty in open-channel discharges measured with the velocity-area method. Flow Measurement and Instrumentation. $26: 18-29$

LE Coz J. (2012) - Expertise de la station hydrométrique de la Turdine à l'Arbresle, au seuil de Gobelette, rapport technique. $14 \mathrm{p}$
Le Coz J., Camenen B., Dramais G., Ferry M., Rosique J.-L., Ribot-Bruno J. (2011) — Contrôle des débits réglementaires. Application de l'article L. 214-18 du Code de l'environnement, Guide pratique Onema/Cemagref. $128 \mathrm{p}$

Le Coz J., Bonnifait L., Thollet F. (2011) — Expertise de la station hydrométrique de la Nartuby à Trans, rapport technique, $26 \mathrm{p}$

Moyeed R.A., Clarke R.T. (2005) - The use of Bayesian methods for fitting rating curves, with case studies. Advances in Water Resources. $28: 807-818$

Petersen-Overleir A., Reitan T. (2009) - Bayesian analysis of stage-fall-discharge models for gauging stations affected by variable backwater. Hydrological Processes. 23(21) : 3057-3074

Petersen-Overleir A. (2004) - Accounting for heteroscedasticity in rating curve estimates. Journal of Hydrology. 292(1-4) : 173-181

Reitan T., Petersen-Overleir A. (2008) - Bayesian power-law regression with a location parameter, with applications for construction of discharge rating curves. Stochastic Environmental Research and Risk Assessment. 22(3) : 351-365

Reitan T., Petersen-Overleir A. (2009) - Bayesian methods for estimating multi-segment discharge rating curves. Stochastic Environmental Research and Risk Assessment DOI 10.1007/ s00477-008-0248-0. 23(5) : 627-642

Renard B., Garreta V., Lang M. (2006) - An application of Bayesian analysis and Markov chain Monte Carlo methods to the estimation of a regional trend in annual maxima. Water Resources Research. 42, W : 12422

Tolsa M., Aubert Y., Le Coz J., Renard B. (2013) — Méthode de consolidation des courbes de tarage pour les crues d'occurrence rare sur le bassin versant expérimental du Réal Collobrier. Congrès SHF : «Hydrométrie 2013», Paris, 15-16 mai 2013 